sprechung des AGH über weite Strecken den Rang einer veritablen Kommentierung zum AGHV. Das läßt sie von erheblichem Interesse auch für den Praktiker sein, der sich im Zuge fortschreitender auch subregionaler lateinamerikanisch-europäischer Kooperation mit der Rechtsordnung im Andenraum vertraut machen möchte.

Karl-Andreas Hernekamp

\title{
Guido Odendahl
}

\section{Das Recht auf Entwicklung - The Right to Development}

Entstehungsgeschichte, systematische Stellung und Inhalt eines individuellen sowie kollektiven Menschenrechts und Grundprinzips der Völkerrechtsordnung Shaker Verlag, Aachen, 1997, 356 S., DM 89,--

Das Recht auf Entwicklung ist seit dem Vortrag Kéba M'Bayes vor dem International Institute of Human Rights in den 70er Jahren ${ }^{1}$ aus der Diskussion um das Verhältnis von Recht und Entwicklung ${ }^{2}$ nicht mehr fortzudenken. Auch vielfältige Kritik an der Herleitung eines solchen Rechts aus dem Völkervertrags- und Völkergewohnheitsrecht, an seinem Inhalt und seinen Adressaten, schließlich an seiner Durchsetzung haben es nicht vermocht, diesem Nomos seine zentrale Bedeutung für die Formulierung der rechtlichen Anforderungen an die Ordnung der internationalen Gemeinschaft streitig zu machen. Die Vielfalt von Diskussionsbeiträgen ${ }^{3}$ über einen geraumen Zeitraum hinweg macht es erforderlich, gelegentlich im Rahmen einer Bestandsanalyse eine erneute Standortbestimmung vorzunehmen. Die vorliegende Arbeit, eine von Professor Meinhard Schröder betreute Trierer Dissertation, wird ihrem Anspruch einer solchen Standortbestimmung und ihrem - doch recht umfänglichen - Untertitel weitgehend gerecht. Sie schildert eingehend die Entstehung des Rechts auf Entwicklung in seinen vielfältigen Ausprägungen mit seinen Ursprüngen im

Kéba M'Baye, Le droit au développement comme un droit de l'homme, Revue des droits de l'homme 1972, S. 502.

2 Vgl. nur Ernst Ulrich Petersmann, Völkerrecht und Entwicklungshilfe, VRÜ 5 (1972), S. 161, Rainer Tetzlaff, Die Forderungen der Entwicklungsländer nach einer ,neuen, Weltwirtschaftsordnung, VRÜ 9 (1976), S. 33, Hermann Weber, Der Anspruch auf Entwicklungshilfe und die Veränderungen des internationalen Wirtschaftsrechts, VRÜ 11 (1978), S. 5, Albert Bleckmann, Anspruch auf Entwicklungshilfe, VRÜ 12 (1979), S. 5,

3 Die Diskussion fand auch in dieser Zeitschrift ihren Niederschlag. Vgl. - außer den Nachweisen in Fn. 1 - vor allem Philip Kunig, Die "innere Dimension" des Rechts auf Entwicklung - Rechtspolitische Überlegungen zur Inpflichtnahme von Entwicklungsländern, VRÜ 19 (1986), S. 383 und Maria M. Kenig-Witkowska, The Paradigm of Development in International Law, Beiheft 14 zu VRÜ, Baden-Baden 1988, S. 78 ff., sowie die Buchrezensionen in VRÜ 12 (1979), S. 5; 20 (1987), 286; 21 (1988), S. 512 und 22 (1989), S. 519. 
Entwicklungsvölkerrecht. Folgerichtig beschäftigt sich Odendahl zunächst kurz mit Kolonialzeit und Völkerbund als untrennbarer Vorgeschichte der Entwicklungsproblematik. Innerhalb dieser Darstellung der Grundlagen werden auch die für die Entwicklungsproblematik wichtigsten Einrichtungen des Systems der Vereinten Nationen mit Aufgaben und Befugnissen vorgestellt (S. 17 ff.) und deren erste Maßnahmen beschrieben (S. 38 ff.).

Seinen ersten Schwerpunkt legt Odendahl auf die Genese, die wesentlichen Quellen und Grundprinzipien des Entwicklungsvölkerrechts, das aus dem die ersten Dekaden der Vereinten Nationen dominierenden Topos der Entwicklungsproblematik hervorging (S. 47 ff.). Als zentralen Teil des Entwicklungsvölkerrechts behandelt der Verfasser anschließend, in sorgfältiger Abgrenzung der Inhalte der beiden Bereiche, die Neue Weltwirtschaftsordnung (S. 83 ff.) von der anfänglichen grundsätzlichen Ablehnung vieler Staaten zur Ausprägung im Rahmen des Systems der Vereinten Nationen und später auch außerhalb anerkannter zunächst elementarer Grundprinzipien. Diese Darstellung stellt - wie auch die vorangehenden Grundlagen - eine klares und gut leserliches Fundament für die Einordnung des Rechts auf Entwicklung in das Geflecht rechtlicher und außerrechtlicher Instrumentarien dar. Gerade angesichts der häufig beschworenen Gefahr der bloßen, sich gleichsam verselbständigenden Rhetorik im Entwicklungsvölkerrecht ist es von nicht unerheblicher Bedeutung, vor der Behandlung des schon in seiner Begrifflichkeit verwirrenden Rechts auf Entwicklung die verschiedenen Ebenen des Problems und die unterschiedlichen, teils parallelen historischen Entwicklungslinien verwandter Bereiche offenzulegen.

Ein über Jahre hinweg so stark von inoffiziellen Dokumenten, Entschließungen, Deklarationen, Reden, Berichten, Empfehlungen und Stellungnahmen einerseits und Völkerrechtsnormen auf verschiedenen Ebenen andererseits geformtes und bestimmtes rechtliches Konzept kann - sofern man dessen Existenz nicht unterstellt - in seiner Entwicklung ertragbringend möglicherweise nur in zwei Darstellungsschritten erfaßt werden, einem ersten Schritt, der die historischen Abfolge politischer und rechtlicher Fakten und Dokumente ungeachtet der rechtlichen Relevanz schildert (S. 111 ff.), und einem zweiten Schritt, der sich der Verfestigung zu einem Recht auf Entwicklung im Völkerrecht widmet (S. 155 ff.). Odendahl geht diesen Weg einer doppelten Darstellung, die dem Leser anschaulich die wesentlichen Inhalte der im Original kaum eingängig verfaßten Dokumente bis in die jüngste Zeit nahebringt. Erfreulich ist hier auch die Befassung mit den Ergebnissen der UNCED 1992 in Rio de Janeiro (S. 140 ff.).

Die Behandlung von Herleitung, Natur und Inhalt des Rechts auf Entwicklung (S. 155 ff.) kann damit schon auf diesen Grundlagen aufbauen. Odendahl lehnt im Ergebnis die Ableitung des Rechts auf Entwicklung aus der in der Charta verankerten Staatenpflicht zur Zusammenarbeit ab (S. 160). Demgegenüber sieht er die Gesamtheit der Menschenrechtskodifikationen als tragfähigen völkerrechtlichen Geltungsgrund für ein menschenrechtlich geprägtes Recht auf Entwicklung; dieses gehe jedoch nicht über den Gehalt der menschenrechtlichen Normierungen hinaus (S. 163 f.). Die Ableitung eines Rechts auf Entwicklung als allgemeinen Rechtsgrundsatz aus nationalen Rechtsinstituten lehnt Odendahl ab (S. 164 f.). Für den Nachweis völkergewohnheitsrechtlicher Geltung verfolgt der Verfasser den 
Weg der Ableitung aus Grundprinzipien der Völkerrechtsordnung einerseits (S. 166 ff.) und des Nachweises der Geltung als aus Völkergewohnheitsrecht hervorgegangenem eigenständigem Verfassungsprinzip des Völkerrechts andererseits (S. 185 ff.). Aus den untersuchten Grundprinzipien favorisiert Odendahl - in leider nur groben Zügen - das Selbstbestimmungsrecht der Völker als lex generalis. Hinsichtlich des Nachweises als Völkergewohnheitsrecht vertieft der Verfasser erneut die Vielfalt der schon angesprochenen Deklarationen und Resolutionen internationaler Organisationen, hier als Hilfsmittel zum Nachweis einer bestehenden opinio iuris. Fast lapidar verweist Odendahl für die Staatenpraxis auf die Tätigkeit der zuvor behandelten Institutionen und nicht näher benannte bilaterale Verträge sowie die Summe internationaler Entwicklungshilfeleistungen (S. 191). Da er hiermit den Nachweis einer der gesicherten Rechtsquellenlehre entsprechenden, von Rechtsüberzeugung getragenen, hinreichend dauerhaften Staatenpraxis durch erneuten Verweis auf die Arbeit internationaler Organisationen ersetzt und vor allem den Nachweis der Konnexität zwischen in den Verlautbarungen der internationalen Organisationen formulierten Verpflichtungen und den dezentral, überwiegend bilateral erfolgenden Leistungen schuldig bleibt, setzt sich der Verfasser ungeachtet der sorgfältigen Vorarbeit in Hinblick auf das Völkergewohnheitsrecht dem Vorwurf unvollständiger rechtlicher Analyse aus.

Das von ihm insoweit festgestellte Recht auf Entwicklung ordnet Odendahl anschließend mit M'Baye als sowohl kollektives wie auch individuelles Menschenrecht ein (S. 194 ff.) und setzt es ins Verhältnis zu den traditionellen Menschenrechten und den sonstigen Drittgenerationsrechten (S. 205, 214).

Hinsichtlich des materiellen Gehalts des Rechts auf Entwicklung ordnet Odendahl seine Überlegungen in die der Grundrechtsdogmatik des deutschen Verfassungsrecht entliehenen Kategorien der Adressaten (S. 217), des Schutzbereichs (S. 236) und der Schranken des Rechts (S. 281). So sehr die Völkerrechtsordnung zu einer analogen Heranziehung von Kategorien des nationalen Verfassungsrechts einlädt, bleibt Odendahl dem Lesẹ die völkerrechtsdogmatische Untermauerung seines Vorgehens schuldig. Dies erweist sich an einigen Stellen als Manko, wenn etwa von "immanenten Schranken kollidierender Rechtspositionen" die Rede ist (S. 281), die angedachte Abwägung jedoch nicht in den dogmatischen Zusammenhang einer angenommenen homogenen Gesamtrechtsordnung gestellt wird. Ungeachtet der mangelnden dogmatischen Einordnung in die Völkerrechtsordnung und der Gefahr der Gleichsetzung mit der Dogmatik des innerstaatlichen Verfassungsrechts mag mit dieser Vorgehensweise jedoch ein Gewinn an Klarheit der Darstellung für den in Begriffen des deutschen Verfassungsrechts denkenden Leser verbunden sein.

Im Rahmen der Darstellung des materiellen Gehalts des Rechts auf Entwicklung unterscheidet Odendahl zunächst in Anknüpfung an den Stellenwert des von ihm festgestellten Rechtssatzes und die Literatur zwischen der inneren und der äußeren Dimension (S. 219) ${ }^{4}$. Er unterscheidet jedoch nicht zwischen einer primären (nach innen gerichteten) Rechts-

Vgl. hierzu Kunig in VRÜ 19 (1986), S. 383. 
pflicht und einer daraus folgenden äußeren Sekundärverpflichtung zur Unterstützung dieses Prozesses durch andere Staaten. Daher rührt seine Behandlung aller Normadressaten in einem einheitlichen Zusammenhang, beginnend mit den Staaten. Dabei lehnt er sowohl eine Sonderstellung von unterentwickelten Staaten wie von Schwellenländern ab. Internationale Organisationen schieden als Adressaten aus (S. 229). Völkern, verstanden als eigenständigem, vom Staatsvolk zu trennenden Begriff, komme die Adressatenstellung ebenfalls zu, eine Verpflichtetenstellung jedoch nicht gegenüber Staaten, sondern nur gegenüber anderen Völkern und Individuen. Im Rahmen der Behandlung der Adressatenstellung von Individuen wird, wie an verschiedenen anderen Stellen zuvor, der Nachteil der gewählten Aufgliederung deutlich: Die Adressatenstellung als Berechtigter bzw. Verpflichteter ist bezogen auf das jeweilige Verständnis des Norminhalts. Die Nutzbarmachung der vorab eingeführten Unterscheidung in eine äußere und eine innere Dimension hätte das immanente Dilemma der Darstellung beseitigt.

Maßgeblich für die Bestimmung des materiellen Inhalt des Rechts ist für Odendahl ein dynamischer Begriff der Entwicklung (S. 251), der auch die Gewährleistung anderer grundlegender Menschenrechte sowie von Partizipation beinhaltet (S. 254 ff.) und durch Umweltschutzerfordernisse beschränkt ist (S. $264 \mathrm{ff}$.).

Nachdem Odendahl die menschenrechtliche Kategorie in dieser Weise erörtert hat, greift er in seiner Zusammenfassung des materiellen Gehalts unvermittelt erneut unterstützend auch auf den Rechtscharakter als Grundprinzip der Völkerrechtsordnung (S. 262) zurück, ohne daß ersichtlich wird, wieso es in den vorangeganenen Ausführungen ohne Bedeutung war. Im Ergebnis hält er den materiellen Gehalt nur beschränkt für konkretisierbar. Der Ertrag lasse sich in ein Abwehrrecht gegen entwicklungshemmende Eingriffe, ein Leistungsrecht auf staatliche Entwicklungsförderung und ein Recht auf aktive demokratische Teilhabe an der Gestaltung des individuellen und kollektiven Entwicklungsprozesses aufgliedern (S. 263). Dies nicht verstanden als Ansprüche zur Durchführung von Einzelmaßnahmen, sondern versehen mit einem weiten, nicht abschließend normierten Freiraum (Konkretisierungsmacht).

$\mathrm{Zu}$ beklagen ist, daß der Beitrag die präferenzielle Behandlung von Entwicklungsländern nur im Rahmen der Darstellung des Konzepts der Neuen Weltwirtschaftsordnung (S. 90) anspricht und im übrigen vernachlässigt. Auch die Ausgestaltung des Rechts auf Entwicklung in Entwicklungshilfeaktivitäten internationaler Organisationen wird nicht behandelt.

Das Werk ist aber ein gelungener Versuch einer eigenständigen Darstellung, die - nicht immer der Völkerrechtsdogmatik folgend - die Rhetorik des Rechts auf Entwicklung auf den rechtlichen Gehalt hin untersucht. Hier durch Klarheit und eigenständigen Aufbau zu Widerspruch und Diskussion, möglicherweise im Rahmen einer Diskussion um materielle Inhalte einer (zukünftigen oder abzuleitenden) internationalen Verfassungsordnung zu neuen Einsichten beizutragen, mag man der Arbeit von Odendahl gern bescheinigen.

Das Buch wird abgerundet durch kurze zusammenfassende Thesen auf Deutsch und Englisch, eine Chronologie der wichtigsten Etappen auf dem Weg zum Recht auf Entwicklung, 
einem Verzeichnis von relevanten Dokumenten internationaler Organisationen und einem Schlagwortverzeichnis.

UlfMarzik

\author{
Gilles Boëtsch / Baudouin Dupret / Jean-Noël Ferrié (Hrsg.) \\ Droits et sociétés dans le monde arabe: perspectives socio-anthropologiques \\ Mit einem Vorwort von Norbert Rouland \\ Presses Universitaires d'Aix-Marseille, Aix-en-Provence, 1997, 229 S., 150 FF
}

Der vorliegende Sammelband versteht sich als ein Plädoyer für einen Paradigmenwechsel bei der Beschäftigung mit dem Recht arabisch-islamischer Gesellschaften: Bislang, so Dupret und Ferrié in der Einleitung, habe bei der Auseinandersetzung mit dem islamischen und arabischen Recht viel zu sehr dessen "kulturelle Besonderheit" im Vordergrund gestanden. Dies zeige sich daran, daß als analytische Kategorien in erster Linie von der Islamwissenschaft geprägte Begriffe - wie etwa die "Schließung des Tors des ijtihâd" oder

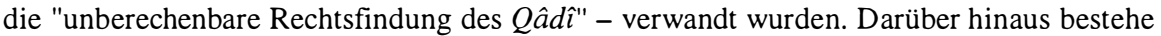
die Tendenz, heutige Entwicklungen in erster Linie historisch, also aus der arabisch-islamischen Geschichte zu erklären. Beides, betonen die Herausgeber, stelle eine Sackgasse dar: Auch das islamische und arabische Recht lasse sich mit Hilfe allgemeiner Kategorien beschreiben. Dies erfordere allerdings eine Abkehr von dem überkommenen, juristischislamwissenschaftlich geprägten Paradigma und eine Öffnung hin zu einem soziologischethnologischen Ansatz.

Die 11 in dem Band veröffentlichten, thematisch und geographisch aus den unterschiedlichsten Bereichen stammenden Beiträge sind daher auch durch einen sozialwissenschaftlichen Ansatz lose verklammert: Die Beschäftigung mit juristischen Fragen ist dem Anspruch nach nicht Selbstzweck, sondern Recht ist Gegenstand historischer und soziologischethnologischer Forschung. Den Anfang macht ein Beitrag von Paradelle, der die klassische islamische Völkerrechtslehre aus einer soziologischen Perspektive nachzeichnet. Es folgen zwei Beiträge zur neueren ägyptischen Rechtsgeschichte: Goldberg geht der Frage nach, wieso Ägypten, seit 1882 unter britischer Herrschaft, dennoch französisches Recht rezipiert hat, und Afifi untersucht die Reaktion der Kopten auf die Reformen des Familienrechts in den zwanziger Jahren. Zwei Beiträge zum marokkanischen Familienrecht schließen sich an: Buskens setzt sich mit dem Prozeß der Redaktion und Kodifikation des islamischen Familienrechts auseinander, Rosen resümiert 30 Jahre rechtsethnologische Feldforschung am Familiengericht Sefrou. Stärker theoretisch ausgerichtet sind die beiden folgenden Beiträge: Boëtsch und Ferrié kritisieren hier den überkommenen Gegensatz von "Norm" und "Praxis", Ben Néfissa den "dogmatischen", autoritätsfixierten Rechtsbegriff. Lebon leitet 